\title{
Research and Analysis of Structural Hole and Matching Coefficient
}

\author{
Penghua Cai, Hai Zhao, Hong Liu, Rong Pan, Zheng Liu, Hui Li
}

Department of Information Science and Engineering, Northeast University, Shenyang, China.

Email: caipenghua2008@yahoo.cn

Received August $28^{\text {th }}, 2010$; revised September $18^{\text {th }}$, accepted September $23^{\text {rd }}, 2010$.

\begin{abstract}
Measure is a map from the reality or experimental world to the mathematical world, through which people can more easily understand the properties of entities and the relationship between them. But the traditional software measurement methods have been unable to effectively measure this large-scale software. Therefore, trustworthy measurement gives an accurate measurement to these emerging features, providing valuable perspectives and different research dimensions to understand software systems. The paper introduces the complex network theory to software measurement methods and proposes a statistical measurement methodology. First we study the basic parameters of the complex network, and then introduce two new measurement parameters: structural holes, matching coefficient.
\end{abstract}

Keywords: Large-Scale Software, Trustworthy Measurement, Structural Holes, Matching Coefficient

\section{Introduction}

Now large software network is increasingly showing "small world" and "scale-free"-characteristics of complex networks. The results of studying complex networks provide strong support for people to explore characteristics of the overall structure of large-scale software network [1,2]. Using a network view research the software network, this has been recognized by more and more researchers. The traditional measurement methods focus on the micro-level statistics and only do some aspects of the software evaluation because of lacking parameters. Therefore, the paper imports complex network theory into the traditional measurement methods and introduces some new metrics to measure the different characteristics of the software from the different levels. This paper also puts forward a measurement methodology, which make the basic intrinsic property and the overall measures properties of software as the core and use multiple measurement parameters (the basic parameters in complex network, the newly introduced metric) to measure some important characteristics and structural features, providing an important basis for measuring software quality.

\section{Structural Hole}

1) The theory of structural hole

The concept of structural holes is from the social structure of competition [3]. It is form social network research. In brief, structural holes are the relationship between the two non-duplicate persons. In Figure 1, we use software network formed by four nodes A, B, C, D to illustrate structural hole. In the left picture A has three structural holes (BC, BD and $\mathrm{CD}$ ); because the three nodes $\mathrm{B}, \mathrm{C}, \mathrm{D}$ have no direct connection and only node A is associated with these three classes. Compared with other three nodes, node A has competitive advantage. It is in the center, so most likely close to all the nodes in the software network. The right picture is actually a closed network, so there is no structural hole.

Figure 1 shows two extreme cases of structural hole in the small-scale software network: the whole-hole structure network and no-hole structure network. In the actual software, it has three types of structure as following:
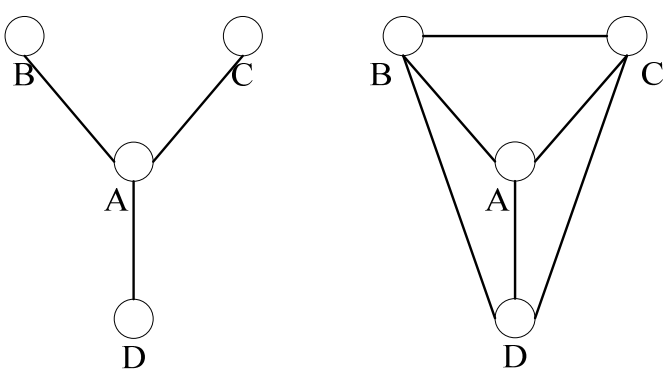

Figure 1. Examples of structural holes. 
Any node in the software network has direct contact with other nodes. From the whole network view, it is "no-hole" structure. This structure only exists in smallscale software network and such groups are actually closed, so the importance of each node in the networks is basically equal. There are many nodes needed to be updated. It is difficult to control them and update software. In addition, the cost of maintaining this high redundancy network is high.

Only the central node has direct link with every other node in the network. The other nodes do not connect with every node directly. From the whole view of the network, the phenomenon of no direct contact or relationship breaking off is structural holes. There are no direct connections among the rest nodes, which is whole-hole structure.

2) The algorithm of structural holes

In the aspects of structural holes measurement, structural constraint algorithm and betweenness centrality algorithm have been used. Structural constraint algorithm uses closeness among nodes as measure targets, dependence among nodes as the evaluation criteria. It can determine the degree of software network structural holes.

At the same time if nodes across more structural holes, they have less redundant connections, can access more non-redundant information and are used more frequently. Betweenness centrality algorithm largely determines the centering level of the nodes. Therefore, the paper uses structural constraint algorithm to compute structural holes.

Definition 2.1 Network Constraint index: This index describes direct or indirect closeness between a node and other nodes. If the network Constraint index is higher, the network is closer and the structural holes are fewer. The concrete calculating steps is as follows [4]:

$$
p_{i j}=\frac{d_{i j}+d_{j i}}{\sum_{k}\left(d_{i k}+d_{k i}\right)}
$$

$p_{i j}$ is the ratio of the shortest path length between node $i$ and node $j$ to the sum of the shortest path length about all the neighboring nodes of node $i . d_{i j}$ is the shortest path length between node $i$ and node $j$.

$$
c_{i j}=\left(p_{i j}+\sum_{k, k \neq i, k \neq j} p_{i k} p_{k j}\right)^{2}
$$

$c_{i j}$ is the binding level between node $i$ and node $j$. When node $j$ is the only adjacent node of node $i, c_{i j}$ gets maximal value 1 . When node $j$ is indirectly connected with node $i$ through other nodes, $c_{i j}$ gets minimum value ${p_{i j}}^{2}$. Node $\mathrm{k}$ is the adjacent node of node $i$.

By formula (2) and formula (3) we can calculate network constraint index of node $i$.

$$
C_{i}=\sum_{j} C_{i j}
$$

Structural holes are used to describe a node in dependence on other nodes. Few structural holes show strong dependence on other nodes. Network constraint index is the quantization of structural holes. By calculating the network constraint index of structural holes, we can understand the degree of structural holes in the software network.

\section{Matching Coefficient}

In 2002, Newman put forward another important statistical parameter used to mark the network, which is assortativity. Assortativity is represented by $r$. It is changing between -1 and 1 that means nodes are prior to establish side connection with similar nodes in the network $[5,6]$. When $r$ is greater than zero, nodes are prior to connect with similar nodes. Such network is called assortative mixing. When $r$ is less than zero, nodes are prior to connect with dissimilar nodes. Such network is called disassortative mixing.

Definition 3.1 assortative coefficient: Incidence relation between nodes in the network can be described by assortative coefficient $[7,8]$ :

$$
r=\frac{E^{-1} \sum_{i} j_{i} k_{i}-\left[E^{-1} \sum_{i} \frac{1}{2}\left(j_{i}+k_{i}\right)\right]^{2}}{E^{-1} \sum_{i} \frac{1}{2}\left(j_{i}^{2}+k_{i}^{2}\right)-\left[E^{-1} \sum_{i} \frac{1}{2}\left(j_{i}+k_{i}\right)\right]^{2}}
$$

$j_{i}$ and $k_{i}$ are the degree of the $i$ side's two vertices. $E$ is the number of sides in the network.

If assortative coefficient is greater than 0 , the network is assortative mixing; if assortative coefficient is less than 0 , the network is disassortative mixing; if assortative coefficient is equal to 0 , the network is randomized. Assortative coefficient reflects the connectivity of network nodes. In the assortative mixing network, nodes of a high degree tend to connect with nodes of a high degree. In the disassortative mixing network, nodes of a high degree tend to connect with nodes of a low degree. In Figure 2, it is a network composed by 10 nodes. In Figure 2(a), $r=-0.372881$. Node 1 's degree is 5, which is a high degree node and connect with nodes (degree is 2 or 1 ). Such network is disassortative mixing. In Figure 2(b), $r=1$. Degrees of all nodes are similar, that is assortative mixing.

\section{The Law and Analysis of Metrics in the Network Software}

\subsection{Correlation Analysis of Degree and Structural Holes}

Degree is used to describe the connected complexity of a 


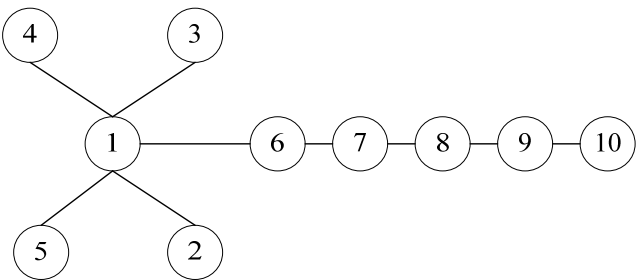

(a)

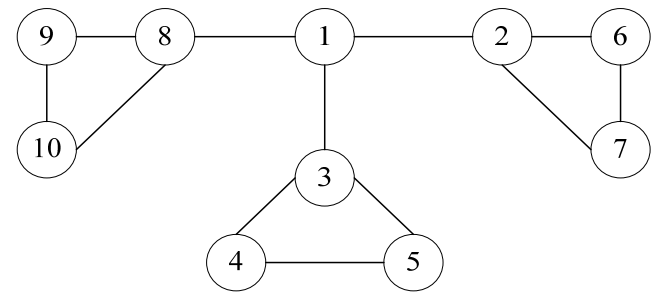

(b)

Figure 2. Examples of assortative mixing and disassortative mixing.

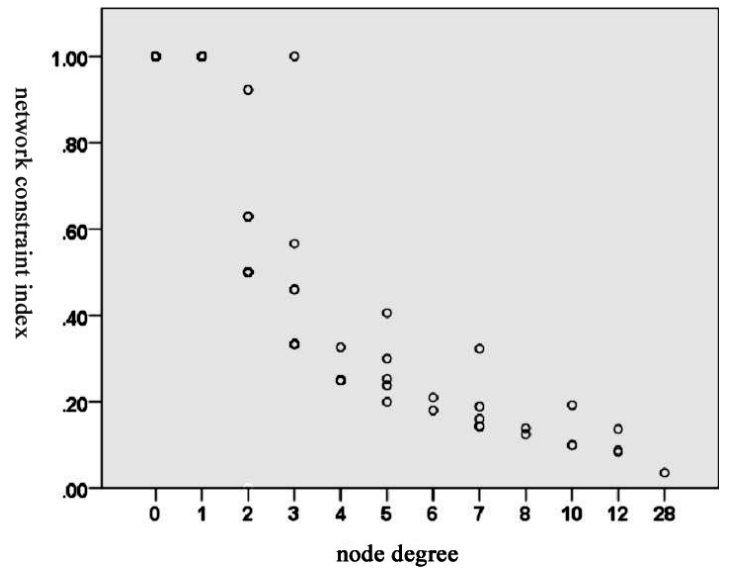

(a) Quartz

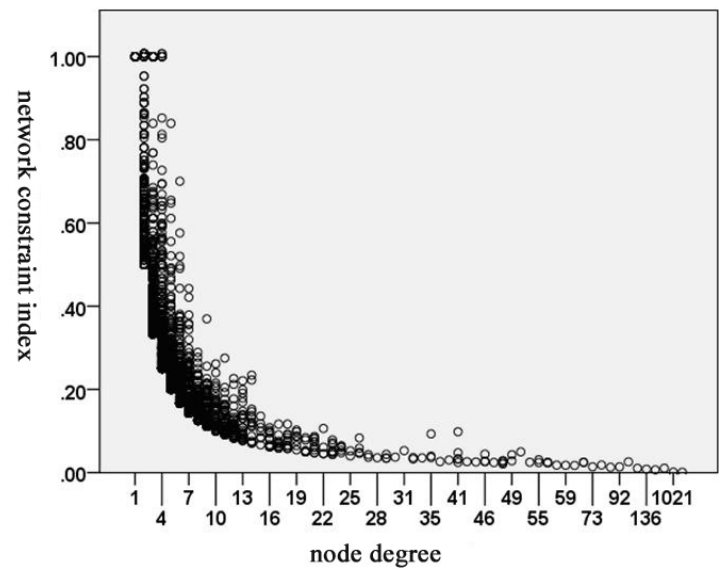

(c) Mozilla
Table 1. The statistical characteristics of 4 kinds of software network.

\begin{tabular}{ccccc}
\hline $\begin{array}{c}\text { Software } \\
\text { system }\end{array}$ & $\begin{array}{c}\text { number of } \\
\text { nodes }\end{array}$ & $\begin{array}{c}\text { isolated } \\
\text { nodes }\end{array}$ & $\begin{array}{c}\text { number of } \\
\text { edges }\end{array}$ & $\begin{array}{c}\text { average } \\
\text { degree }\end{array}$ \\
\hline Quartz & 255 & 63 & 231 & 1.81176 \\
Abiword & 1712 & 203 & 2484 & 2.84211 \\
Mozilla & 8354 & 1159 & 13581 & 3.32248 \\
Eclipse & 14730 & 1721 & 27560 & 3.74202 \\
\hline
\end{tabular}

node and its neighboring nodes. The larger value of a node degree, the more important it shows, but not for chain network. Structural holes are used to show the importance of a node from another point.

First we analyze the network structure of four object-oriented networks (Quartz, Abiword, Mozilla and Eclipse), as shown in Table1. As can be seen from Table $\mathbf{1}$, the scales of them vary widely. Compared with total nodes, isolated nodes were few. So the four software

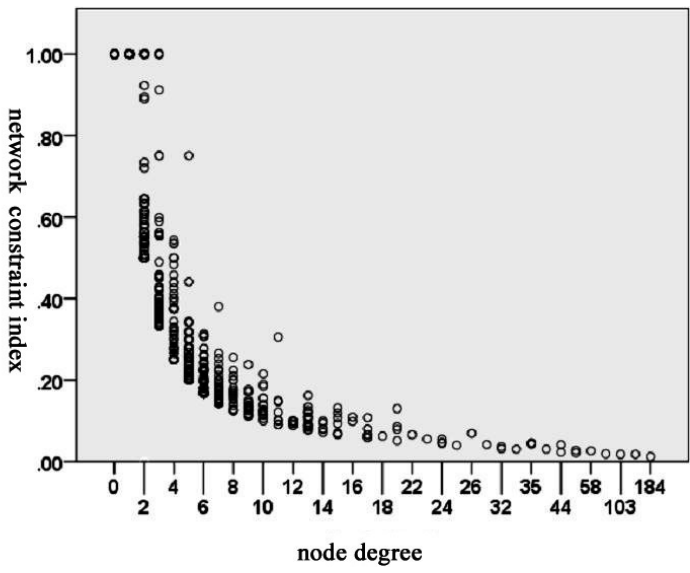

(b) Abiword

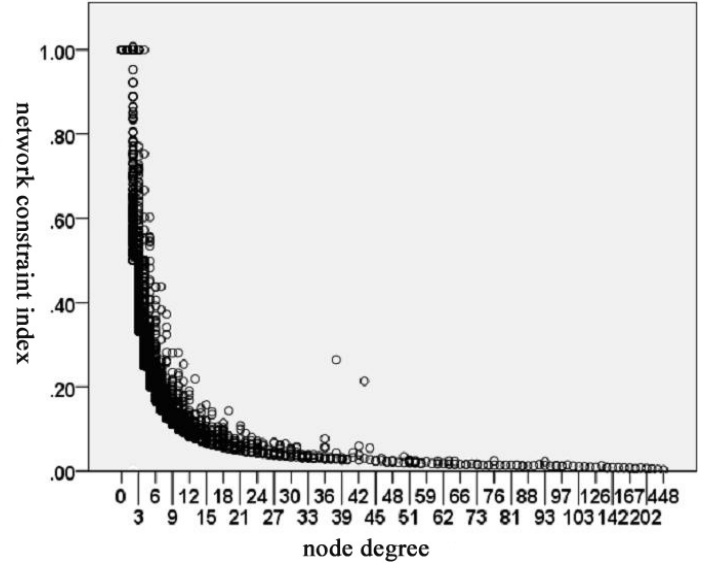

(d) Eclipse

Figure 3. Diagram of the distribution of network constraint index and degree. 
networks have representativeness in all software samples.

The paper analyzes interdependency of degree and structural holes about these four software network. As the structural holes are quantified through network constraint index, so interdependency of degree and structural holes is also interdependency of degree and network constraint index. In Figure 3, horizontal ordinate is the value of every node's degree, vertical coordinates is the value of network constraint index. In all software network, the greater the value of nodes degree, the smaller network constraint index, the more structural holes, the weaker dependency on the around nodes. A special case is that a node's degree is 0 and its network constraint index is 1 , then the node does not have structural holes. It is isolated node. In the software network it will not be called by other operations.

Since isolated nodes do not affect the software feature, after removing isolated nodes we make curve fitting to the relationship of degree and network constraint index. In Figure 4, horizontal ordinate is the value of node's

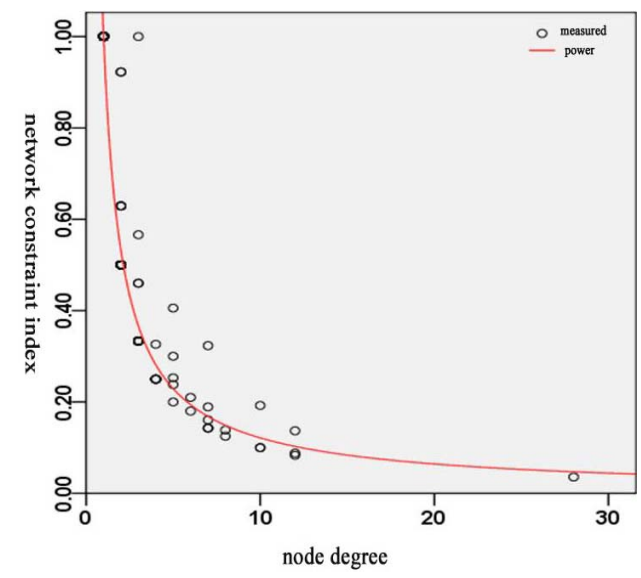

(a) Quartz

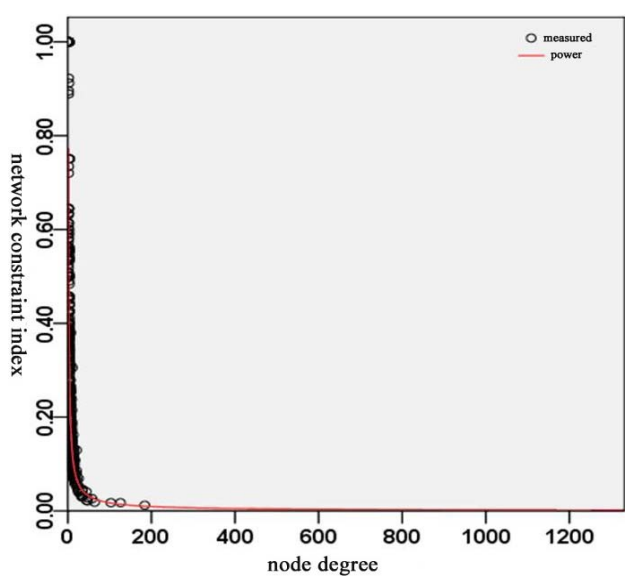

(c) Mozilla degree, vertical coordinates is the value of network constraint index.

Relationship distribution curve of structural holes and network constraint index is power curve, which shows an important feature of software system modularization. Fitting curve is the mathematical expression of this feature. For example, Software Network Quartz's fitted power function equation is as follows:

$$
Y=1.003 \times X^{-0.918}
$$

$X$ is the nodes' degree value (abscissa). These four software network' parameter estimates are shown in Table 2. In software network, the greater the value of nodes degree, the smaller network constraint index, the more structural holes.

Whether a regression model is good or not, the most commonly used index is the coefficient of determination $[9,10]$. The index is based on the decomposition of the dispersion quadratic sum. Coefficient of determination is a comprehensive measure for regression model's goodness of fit $[11,12]$.

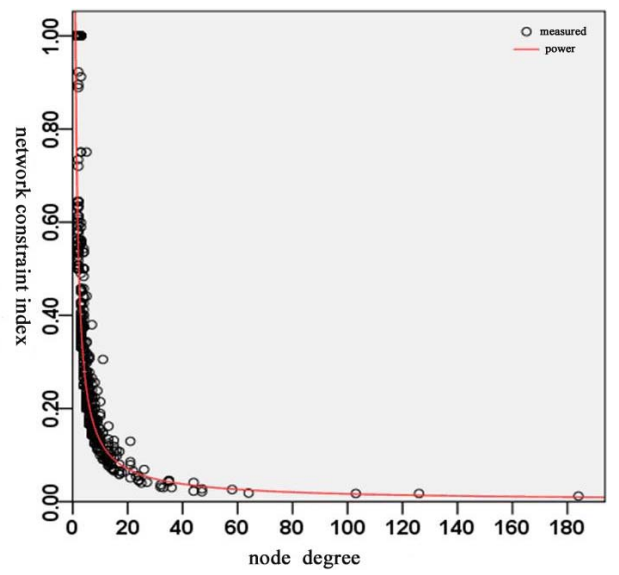

(b) Abiword

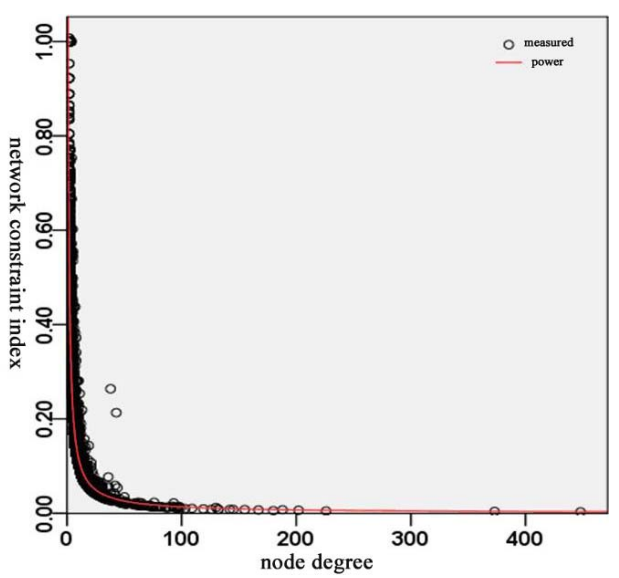

(d) Eclipse

Figure 4. The fitness graph of relationship between network constraint index and degree. 
Table 2. Model summary and parameter estimation.

\begin{tabular}{ccccccc}
\hline \multirow{2}{*}{$\begin{array}{c}\text { Software } \\
\text { system }\end{array}$} & \multicolumn{3}{c}{ model summary } & & \multicolumn{2}{c}{ parameter estimate } \\
\cline { 2 - 4 } \cline { 6 - 7 } & $\mathrm{R}$ & $\mathrm{F}$ & Sig. & & constant & $\mathrm{b} 1$ \\
\hline Quartz & .955 & 3995.527 & .000 & & 1.003 & -918 \\
Abiword & .943 & 24989.658 & .000 & & 1.016 & -892 \\
Mozilla & .917 & 79097.785 & .000 & & .996 & -882 \\
Eclipse & .960 & 313520.018 & .000 & & 1.004 & -934 \\
\hline
\end{tabular}

Formula of correlation coefficient:

$$
r=\frac{N \sum X Y-\sum X \sum Y}{\sqrt{N \sum X^{2}-\left(\sum X\right)^{2}} \sqrt{N \sum Y^{2}-\left(\sum Y\right)^{2}}}
$$

Formula of determination coefficient:

$$
R=r^{2}
$$

F test is mainly for variance analysis. Sig is result of $F$ test. If Sig is less-than 0.05, which declare that difference is significant.

From Table 2 the coefficient of determination $R=$ 0.958 , Sig $<0.05$. Therefore we can conclude goodness of fit is very high and fitting power function can fully reflect a power curve relationship between network constraint index and node degree. So fitting results is acceptable.

Through the four software networks we can see that the structural holes obey specified rule. Enlarge sample, and then test 200 software networks. The results are shown in Figure 5. Abscissa is the software serial number. In Figure 5 vertical coordinates is the goodness of fit; in Figure 5(b) vertical coordinates is the relation fitting power function curve parameter estimates of network constraint index and degree.

In Figure 5(a), goodness of fit of the network constraint index and the degree is between 0.80 and 0.98 . This shows that relationship of the network constraint index and the degree apparently obeys power function distribution. Of course, 25 software networks' goodness of fit is between 0.50 and 0.80 , which indicate the network constraint index and the degree are moderate correlation. In addition, 3 software networks' goodness of fit is less than 0.5 , which indicate the network constraint index and the degree are low correlation. In Figure 5(b), power function relation of network constraint index and degree changes little.

In software network, correlation of degree and structural holes contributes to analyze collaborative relationships between different types of software entities. It is useful to discover software entities' problems. Complex class or module are tend to be composed by relatively simple class or module. This is the software constructivity principle. On the other hand, correlation between the network constraint index and the degree of structural

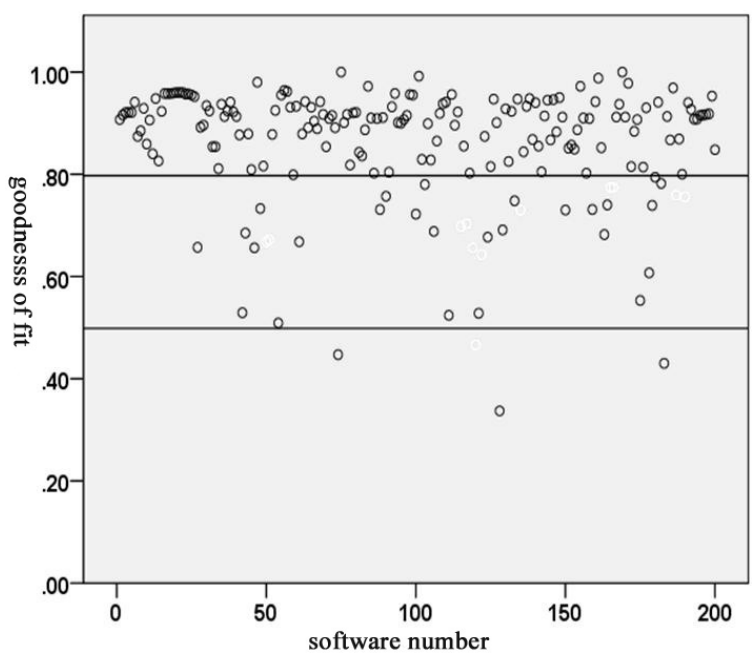

(a)

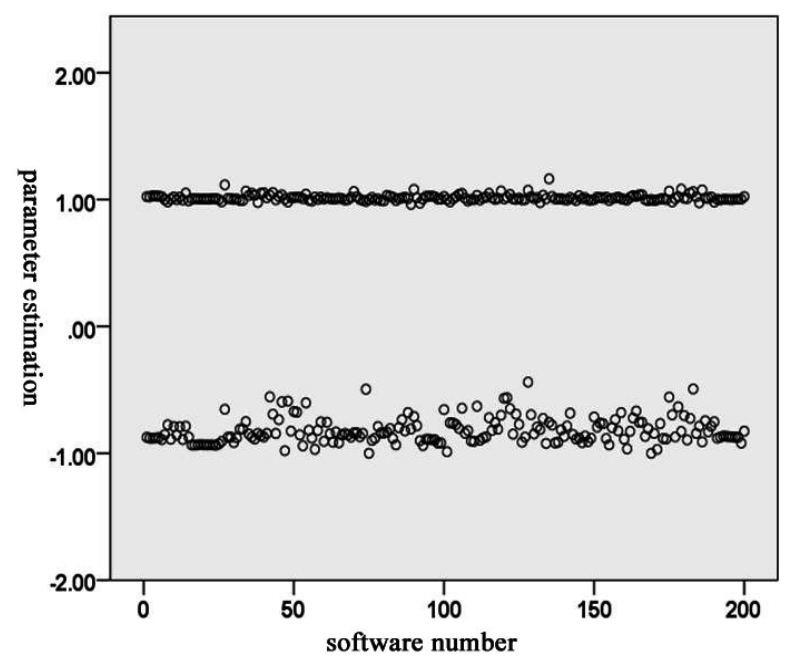

(b)

Figure 5. Diagram of the distribution coefficient of etermination and parameter estimation.

holes is helpful to the analysis of system hierarchy and modularity. Class or module with large degree are tend to gather with class or module with small degree, that shows a high cohesion

\subsection{Law of Matching Coefficient}

The paper makes a further analysis on the 200 samples and calculates the matching coefficient for each software network. The results are shown in Figure 6. In the 200 software networks, $80 \%$ of them are disassortative mixing; $20 \%$ of them are assortative mixing.

First of all, we analyze the disassortative mixing software network, because they occupy majority of the software samples. Software network currently in use most are disassortative mixing. From Table 3 we can conclude that disassortative mixing software networks have no 


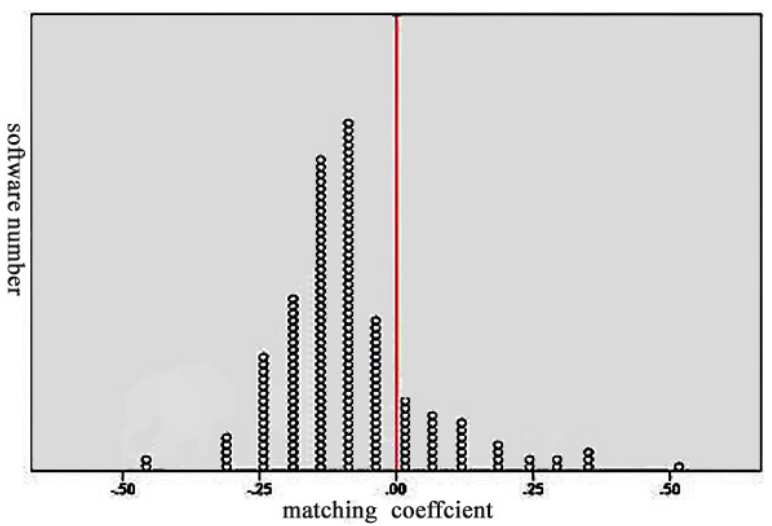

Figure 6. Diagram of the distribution of the mixing coefficient.

concern with the total number of nodes. The average degree, the average structural holes of the disassortative mixing software network don't have obvious law. Some software networks are well known and have higher evaluation. Their coefficient of determination of structural holes and degree are greater than 0.8 .

Some assortative mixing software networks are shown in Table 4. As can be seen from Table 4, assortative mixing software network is different from disassortative mixing software network; moreover network constraint index and degree goodness of fit is relatively low. The number of assortative mixing software network' nodes are generally small, and matching coefficient has nothing to do with the average degree and structural holes.

By comparison, it is discovered that in the 200 software networks, when the total number of nodes is more than 1,000 , they are disassortative mixing software networks. In these software networks, the nodes with lower degree are considered as a relatively simple module in the software network. In disassortative mixing software network, nodes with high degree tend to connect with nodes with low degree. The nodes with lower degree are conducive to the decomposition of software tasks, while the nodes with higher degree are key points for software modules completing the complex task.

Compared Table 3 with Table 4, it can be concluded that matching coefficient was correlated with the number of nodes. The relationship between matching coefficient and software size is shown in Figure 7. The abscissa is the total number of each software network's nodes, the vertical coordinates is the matching coefficient. In Figure 7(a), the number of each software network's nodes is less than 1000. The majority of software network's matching coefficients are below 0. In Figure 7(b), the number of each software network's nodes is greater than 1000 . When the number of nodes is greater than 1000 , the software network is disassortative mixing.

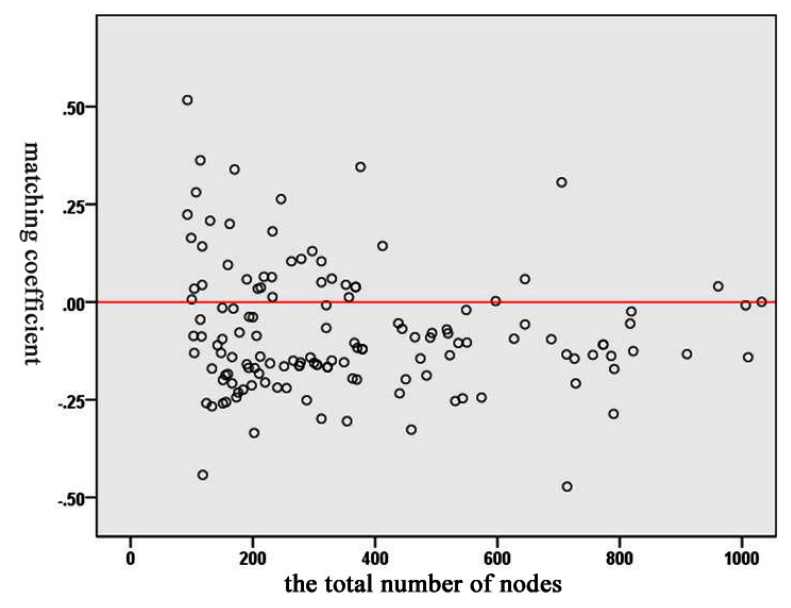

(a) The number is less than 1000 .

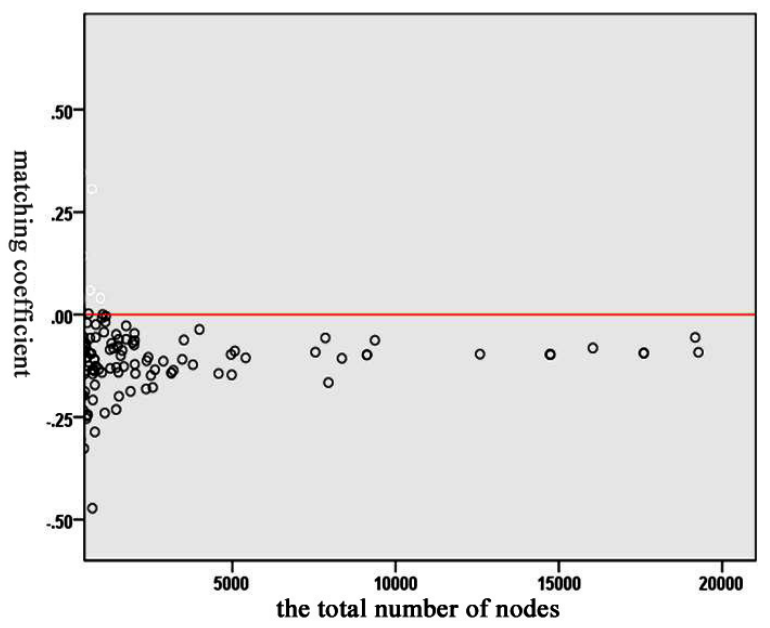

(b) The number is more than 1000 .

Figure 7. Relationship between the mixing coefficient and the number of nodes.

As the pressure of design and implementation, it is necessary to keep each module simple and effective. When a node has a high degree, it also has the features of complexity and high multiplexing. In the assortative mixing software network, if a node has a high degree or connects with high degree nodes that will cause system problems. System maintainability and modifiability fall down. It is need to reconstruct for such modules

\section{Conclusion}

The paper uses structural holes and the matching coefficient to measure software. Software structural holes measure the software network from the software dependent features. Correlation between network constraint index and the degree obey power law distribution that reflects an important software feature. As the study of the software network is still in the exploration stage, so the study of measurement methods of software network 
Table 3. Aisassortative mixing.

\begin{tabular}{lccccc}
\hline \multicolumn{1}{c}{ Software name } & matching coefficient & nodes & goodness of fit & average degree & average structural holes \\
\hline kdegraphics-3.5.3 & -0.121387 & 2014 & 0.931 & 3.32572 & 0.600915 \\
mysql_6.0.6 & -0.12225 & 3793 & 0.836 & 2.83048 & 0.709367 \\
jEditR1.35 & -0.125693 & 822 & 0.868 & 1.74696 & 0.815401 \\
kdebase-3.5.3 & -0.126603 & 1677 & 0.879 & 2.12165 & 0.745993 \\
kdevelop-3.4.0 & -0.128954 & 1453 & 0.917 & 1.97385 & 0.77426 \\
qhacc-3.4 & -0.130487 & 148 & 0.932 & 3.22973 & 0.480221 \\
rpm-4.4.1 & -0.131246 & 1260 & 0.815 & 2.05397 & 0.781209 \\
nss-3.9.2 & -0.133362 & 910 & 0.849 & 3.17363 & 0.667572 \\
freemind0.9.0 & -0.13402 & 713 & 0.877 & 2.61711 & 0.714985 \\
mysql_5.1.26 & -0.136164 & 3194 & 0.843 & 2.57044 & 0.732296 \\
sim-0.9.4 & -0.138038 & 786 & 0.93 & 2.44275 & 0.72691 \\
kicad-20060626 & -0.139725 & 212 & 0.854 & 2.83019 & 0.694856 \\
kopete-0.12.1 & -0.140958 & 1512 & 0.891 & 2.65741 & 0.665513 \\
qtiplot-0.8.2 & -0.141222 & 166 & 0.958 & 1.83133 & 0.750994 \\
kdeedu-3.5.4 & -0.141509 & 1010 & 0.891 & 2.04158 & 0.765379 \\
mysql_5.0.67 & -0.142316 & 3133 & 0.921 & 2.45388 & 0.734618 \\
mysql-5.0.56 & -0.142425 & 3132 & 0.92 & 2.45019 & 0.735107 \\
ArgoUML-0.26.2 & -0.143707 & 2031 & 0.828 & 2.18316 & 0.805827 \\
koffice-1.5.0 & -0.143862 & 4580 & 0.915 & 2.57293 & 0.695893 \\
glib-2.16.5 & -0.144575 & 474 & 0.816 & 1.64979 & 0.828419 \\
\hline
\end{tabular}

Table 4. Assortative mixing.

\begin{tabular}{|c|c|c|c|c|c|}
\hline Software name & matching coefficient & nodes & goodness of fit & average degree & average structural holes \\
\hline gnuplot-4.0.0IDE & 0.516592 & 93 & 0.509 & 2.02151 & 0.717183 \\
\hline exim-4.62 & 0.36244 & 114 & 0.337 & 1.21053 & 0.826957 \\
\hline courier-0.52.2 & 0.345491 & 376 & 0.528 & 2.00532 & 0.724346 \\
\hline freeradius-1.1.0 & 0.33909 & 170 & 0.529 & 1.38824 & 0.855368 \\
\hline maildrop-2.0.2 & 0.280709 & 107 & 0.447 & 1.79439 & 0.790998 \\
\hline ups-3.38 & 0.263252 & 246 & 0.43 & 1.72358 & 0.829059 \\
\hline coreutils-5.2.1 & 0.223548 & 93 & 0.466 & 1.2043 & 0.80448 \\
\hline nedit-5.5 & 0.207817 & 130 & 0.73 & 1.63077 & 0.79206 \\
\hline kdeartwork-3.5.4 & 0.199741 & 162 & 0.668 & 1.2963 & 0.847517 \\
\hline evince-0.5.4 & 0.180686 & 232 & 0.677 & 1.2069 & 0.860548 \\
\hline bash-3.2 & 0.163871 & 99 & 0.524 & 1.51515 & 0.797375 \\
\hline electric-7.00 & 0.143483 & 412 & 0.657 & 3.35437 & 0.635638 \\
\hline bibletime-1.6 & 0.0948479 & 159 & 0.896 & 2.03774 & 0.712981 \\
\hline freeradius-2.0.5 & 0.064476 & 218 & 0.685 & 1.6055 & 0.823288 \\
\hline strongswan-2.6.4 & 0.050583 & 312 & 0.722 & 1.69872 & 0.814492 \\
\hline evms-2.5.5 & 0.0442493 & 352 & 0.815 & 1.79545 & 0.790988 \\
\hline jabberd-2.0s11 & 0.0435754 & 117 & 0.73 & 3.65812 & 0.637675 \\
\hline glibc-2.3.6 & 0.0400406 & 961 & 0.668 & 1.26743 & 0.853633 \\
\hline dasher-4.0.4 & 0.0368399 & 213 & 0.84 & 2.83568 & 0.664322 \\
\hline cyrus-2.3.12 & 0.110243 & 279 & 0.643 & 1.94265 & 0.791312 \\
\hline
\end{tabular}

is also in the exploration stage. This paper studies the single property, association of the property and holistic measure of the software. However, arising deviation is inevitable in the process. As the constraints of time and energy, the samples of this paper's research are still small samples. It is need to enlarge samples. Next we need to further examine the effectiveness of measurement methodology in the actual development project, develop and integrate auxiliary means to guide the actual software development.

\section{REFERENCES}

[1] M. Gaertler and R. J. Mondragon, “Accurately Modeling the Internet Topology,” Physics Review E, Vol. 66, No. 18, 2006, pp. 163-167. 
[2] R. S. Burt, "Structural Holes: The Social Structure of Competition,” Harvard University Press, 1995, PP. 35-38.

[3] S. Abdelwahed, N. Kandasamy and A. Gokhale, "High Confidence Sofware for Cyber-Physical Systems," Proceedings of the 2007 Workshop on Automating Service Quality, Atlanta, 2007, PP. 1-3.

[4] Y. T. Ma, J. X. Chen and J. H. Wu, "Research on the Phenomenon of Software Drift in Software Processes," Proceedings of 8th International Workshop on Principles of Software Evolution, Lisbon, September 2005, pp. 195-198.

[5] M. Alshayeb and W. Li, "An Empirical Validation of Object-Oriented Metrics in Two Different Iterative Software Processes," IEEE Transactions on Software Engineering, Vol. 29, No. 11, November 2003, pp. 10431049.

[6] L. C. Briand, S. Morasca and V. R. Basili, "Property-Based Software Engineering Measurement," IEEE Transactions on Software Engineering, Vol. 22, No. 1, January 1996, pp. 68-86.
[7] S. Furey, "Why We Should Use Function Points," IEEE Software, Vol. 14, No. 2, 1997, pp. 28-30.

[8] M. Arnold and P. Pedross, "Software Size Measurement and Productivity Rating in a Large-Scale Software Development Department," Proceedings of 20th International Conference on Software Engineering, Kyoto, 1998, pp. 503-506.

[9] M. Bauer, "Analysing Software Systems by Using Combinations of Metrics," Proceedings of ECOOP'99 Workshops, Lisbon, 1999, pp. 170-171.

[10] S. R. Chidamber and C. F. Kemerer, "A Metrics Suite for Object-Oriented Design,” IEEE Transactions on Software Engineering, Vol. 20, No. 6, June 1994, pp. 476-493.

[11] F. B. e Abreu, "The MOOD Metrics Set," Proceedings of ECOOP'95 Workshop on Metrics, Aarhus, 1995, pp. 150-152.

[12] N. E. Fenton and M. Neil, "Software Metrics: Successes, Failures and New Directions," Journal of Systems and Software, Vol. 47, No. 2-3, July 1999, pp. 149-157. 